\title{
CLOSED BAIRE SETS ARE (SOMETIMES) ZERO-SETS
}

\author{
W. W. COMFORT ${ }^{1}$
}

\begin{abstract}
It is a theorem essentially due to Paul Halmos $[H, 51 . D]$ that each compact Baire set is a zero-set. Kenneth A. Ross and Karl Stromberg [RS] have shown (a bit more than the fact that) if $X$ is a completely regular Hausdorff space which is locally compact and $\sigma$-compact, then each closed Baire set in $X$ is a zero-set; the same conclusion is known to hold in case $X$ is Lindelö and a $G_{\delta}$ in $\beta X$. In the present paper we prove the following theorem, and we show how the "closed Baire set" theorems of Ross and Stromberg emerge as corollaries: If $X$ is Baire in $\beta X$ and $A$ is a closed Baire set in $X$, then $A$ is a zero-set in $X$. Finally, we indicate how our theorem, and hence those of Ross and Stromberg, can be derived from early and forthcoming work of Frolik.
\end{abstract}

1. Closed Baire sets. For each family $Q$ of sets, the symbol $\tau(Q)$ denotes the smallest family $\mathcal{F}$ of sets containing $a$ for which $\bigcup_{n \in N} A_{n}$ belongs to $\mathcal{F}$ and $\bigcap_{n \in N} A_{n}$ belongs to $\mathcal{F}$ whenever each of the sets $A_{n}$ belongs to $\mathcal{F}$ (here and throughout, the set $N$ is the set of positive integers). The family $\tau(Q)$ can be constructed by hand: one has

$$
\tau(a)=\underset{0 \leq \alpha<\omega_{1}}{\bigcup} a_{\alpha}
$$

where by definition

$$
\begin{aligned}
& \mathfrak{Q}_{0}=Q ; \\
& \mathfrak{Q}_{\alpha}=\left\{\bigcup_{n \in N} A_{n}: A_{n} \in Q_{\alpha-1}\right\} \text { for each odd nonlimit ordinal } \alpha ; \\
& \mathfrak{Q}_{\alpha}=\left\{\bigcap_{n \in N} A_{n}: A_{n} \in Q_{\alpha-1}\right\} \text { for each even nonlimit ordinal } \alpha ; \\
& \mathfrak{Q}_{\alpha}=\bigcup_{\gamma<\alpha} Q_{\gamma} \text { for each limit ordinal } \alpha .
\end{aligned}
$$

(Among the predecessors of a given infinite nonlimit ordinal $\alpha$, there is a largest limit ordinal $\lambda(\alpha)$. Thus $\alpha$ has the form $\alpha=\lambda(\alpha)+n(\alpha)$ for some positive integer $n(\alpha)$, and $\alpha$ is said to be odd or even according as $n(\alpha)$ is odd or even.)

We shall consider only completely regular Hausdorff spaces. For each such space $X$, we denote by $Z(X)$, or by $Z$ when confusion is impossible, the family of zero-sets in $X$. The family $B(X)$ of Baire sets in $X$ is, by definition, the smallest family of subsets of $X$ containing $Z$ and closed under passage to the complement, and to the countable union, and to the countable intersection, of its members.

Received by the editors November 10, 1969.

AMS Subject Classifications. Primary 2810, 5420; Secondary 5440, 5450, 5465.

Key Words and Phrases. Baire set, zero-set, Stone-Čech compactification.

1 The author gratefully acknowledges support received from the National Science Foundation, under grants NSF GP-8357 and NSF GP-18825. 
In the notation of the last paragraph, we have $X \backslash Z \in Z_{1}$ whenever $Z \in Z$, whence it follows in fact that $\tau(Z(X))$ is closed under complementation. Thus $B(X)=\tau(Z(X))$.

The lemma which follows uses the technique introduced by Halmos $[\mathrm{H}, 51 . \mathrm{D}]$; see also $\left[\mathbf{N}_{1}\right.$, Theorem 2.1], and $\left[\mathbf{N}_{2}\right.$, p. 607].

1.1. Lemma. Let $S$ and $T$ be Baire sets in $Y$. Then there is a countable collection $W$ of zero-sets of $Y$, and a continuous mapping $q$ from $Y$ onto some metric space $M$, for which

(a) $S \in \tau$ (W) and $T \in \tau($ W $)$;

(b) $A=q^{-1}(q(A))$ whenever $A \in \tau(W)$.

Proof. Since $\tau Q=U\left\{\tau W: W \subset Q\right.$ and $\left.|W| \leqq \boldsymbol{\aleph}_{0}\right\}$ for each class $a$ of sets, we can find countable subfamilies ${ }^{W^{(1)}}$ and ${ }^{W^{(2)}}$ of $\mathcal{Z}(Y)$ with $S \in \tau\left(W^{(1)}\right)$ and $T \in \tau\left(W^{(2)}\right)$. We write $W^{\prime} W^{(1)} \cup W^{(2)}$, and we write

$$
W=\left\{f_{n}^{-1}(0): n \in N\right\}
$$

with each $f_{n}$ a continuous real-valued function on $Y$ for which $0 \leqq f_{n} \leqq 1$.

Defining $d(x, y)=\sum_{n \in N}\left|f_{n}(x)-f_{n}(y)\right| / 2^{n}$ for $(x, y) \in Y \times Y$, and writing $\bar{y}=\{x \in Y: d(x, y)=0\}$, the desired metric space is the set $M=\{\bar{y}: y \in Y\}$ together with the metric $\bar{d}$ defined by the rule $\bar{d}(\bar{x}, \bar{y})=d(x, y)$.

That the map $q$ defined from $Y$ to $M$ by the rule $q(y)=\bar{y}$ is continuous at a fixed point $y_{0}$ is nearly obvious: Given $\epsilon>0$ there is an integer $m$ for which $\sum_{n>m} 1 / 2^{n}<\epsilon / 2$ and a neighborhood $U$ of $y_{0}$ for which

$$
\left|f_{n}(y)-f_{n}\left(y_{0}\right)\right|<\epsilon / m
$$

whenever $y \in U$ and $1 \leqq n \leqq m$; then

$$
\begin{aligned}
\bar{d}\left(q(y), q\left(y_{0}\right)\right) & =\sum_{1 \leqq n \leqq m}\left|f_{n}(y)-f_{n}\left(y_{0}\right)\right| / 2^{n}+\sum_{n>m}\left|f_{n}(y)-f_{n}\left(y_{0}\right)\right| / 2^{n} \\
& \leqq m \cdot 1 / 2 \cdot \epsilon / m+\epsilon / 2=\epsilon .
\end{aligned}
$$

If $A \in W_{0}=W$ then, because for each pair $(x, y)$ of points in $Y$, we have $q(x)=q(y)$ if and only if $f_{n}(x)=f_{n}(y)$ for each $n$, evidently $A=q^{-1}(q(A))$. Now suppose that $\alpha<\omega_{1}$ and that $B=q^{-1}(q(B))$ whenever $B \in \cup_{\gamma<\alpha} W_{\gamma}$, and let $A \in W_{\alpha}$. If $\alpha$ is a limit ordinal we have $A \in W_{\gamma}$ for some $\gamma<\alpha$, so that $A=q^{-1}(q(A))$; otherwise there are elements $\left\{A_{n}\right\}_{n \in N}$ in $W_{\alpha-1}$ for which $A=\bigcup_{n \in N} A_{n}$ or $A=\bigcap_{n \in N} A_{n}$, and in either case from the inductive hypothesis $A_{n}=q^{-1}(q(A))$ it follows that $A=q^{-1}(q(A))$. 
We are now ready to prove the theorem cited earlier.

1.2. Theorem. Let $X$ be a Baire set in $\beta X$ and let $A$ be a closed Baire set in $X$. Then $A$ is a zero-set in $X$.

Proof. Because each bounded real-valued continuous function on $X$ extends continuously to $\beta X$, there is a Baire set $A^{\prime}$ in $\beta X$ for which $A=A^{\prime} \cap X$; thus $A$ itself is a Baire set in $\beta X$. From the lemma above there is a continuous map $q$ from $\beta X$ onto some metric space $M$ with $A=q^{-1}(q(A))$ and $X=q^{-1}(q(X))$. We set $M^{\prime}=q(X)$, and we denote by $q^{\prime}$ the restriction of $q$ to $X$.

To see that $q^{\prime}(A)$ is closed in $M^{\prime}$, let $m_{\alpha}$ be a net in $q^{\prime}(A)$-say $m_{\alpha}=q^{\prime}\left(x_{\alpha}\right)$ with $x_{\alpha} \in A$-for which $m_{\alpha} \rightarrow m \in M^{\prime}$. Because $\beta X$ is compact some subnet of the net $x_{\alpha}$ in $\beta X$ converges to some point $p$ in $\beta X$. Since $q$ is continuous, we have $q(p)=m$, so $p \in q^{-1}\left(M^{\prime}\right)=q^{-1}(q(X))=X$. Since $A$ is closed in $X$ we have $p \in A$, so indeed $m=q(p) \in q(A)$ and $q(A)$ is closed in $M^{\prime}$.

Choosing any continuous real-valued function $f$ on $M^{\prime}$ for which $q(A)=f^{-1}(0)$, we have (as in [H, 51.D] and elsewhere) $: f \circ q^{\prime}$ is continuous on $X$, and $A=\left(f \circ q^{\prime}\right)^{-1}(0)$.

\subsection{Corollary. Let $A$ be a closed Baire set in $X$, and suppose either} that

(a) $X$ is locally compact and $\sigma$-compact; or

(b) $X$ is locally compact and paracompact; or

(c) $X$ is Lindelöf, and $a G_{\delta}$ in $\beta X$.

Then $A$ is a zero-set in $X$.

Proof. (a) The locally compact, $\sigma$-compact spaces are readily checked to be precisely those spaces $X$ which are cozero-sets in $\beta X[\mathrm{G} \mathrm{J}, 3.11(\mathrm{~b})]$. Each such space is a Baire set in $\beta X$, then, so 1.2 applies.

(b) follows from (a): $X$ can be expressed in the form $X=\mathrm{U}_{\gamma \in \Gamma} X_{\gamma}$, where the sets $X_{\gamma}$ are pairwise disjoint open-and-closed $\sigma$-compact subsets of $X$; according to (a) there is for each $\gamma$ a continuous real-valued function $f_{\gamma}$ on $X_{\gamma}$ for which $A \cap X_{\gamma}=f_{\gamma}^{-1}(0)$; writing $f=U_{\gamma \in \Gamma} f_{\gamma}$, we see that $f$ is continuous on $X$ and that $A=f^{-1}(0)$.

(c) Let the Lindelöf space $X$ be expressed in the form $X=\bigcap_{n \in N} U_{n}$, each $U_{n}$ open in $\beta X$. For each point $x$ in $X$ and each $n$ there is a cozero-set $G(x, n)$ in $\beta X$ with

$$
x \in G(x, n) \subset U_{n} .
$$

For $n$ fixed there are countably many such sets whose union contains 
$X$. Since in any space the countable union of cozero-sets is a cozeroset, we have: for each $n$ a cozero-set $G_{n}$ exists for which $X \subset G_{n} \subset U_{n}$. It follows that $X=\bigcap_{n \in N} G_{n}$, so that $X$ is a Baire set in $\beta X$.

It is readily checked that a space $X$ is locally compact if and only if $X$ is open in $\beta X$. This fact yields the following easy result.

1.4. Proposition. Let $A$ be a closed, Baire set in the locally compact space $X$. Then the following conditions are equivalent:

(a) $A$ is $\sigma$-compact;

(b) $A$ is a cozero-set in $\beta A$;

(c) $A$ is a Baire set in $\beta A$.

Proof. Only the implication (c) $\Rightarrow(a)$ is not obvious. If (c) holds then $A$, being locally compact, is an open Baire set in $\beta A$, hence is a cozero-set in $\beta A$, hence is $\sigma$-compact.

The proposition just given sheds no light on the following question, posed by Ross and Stromberg in [RS]: Must each closed Baire set in a locally compact, normal space be a zero-set? We do not know the answer to this question. The following result is a poor substitute.

1.5. Proposition. Let $X$ be locally compact and normal, and let $A$ be a closed Baire set with a locally compact, paracompact neighborhood in $X$. Then $A$ is a zero-set in $X$.

Proof. This follows from 1.3(b), which coincides with Theorem 1.3 of [RS]. Using the normality hypothesis there are open sets $U$ and $V$, with $V$ paracompact, for which

$$
A \subset U \subset \operatorname{cl}_{X} U \subset V,
$$

and a continuous function $f$ from $V$ to $[0,1]$ with $A=f^{-1}(0)$. Extending $f$ continuously to a function $f^{\prime}$ from $X$ to $[0,1]$ and choosing a continuous function $g$ from $X$ to $[0,1]$ with $g \equiv 0$ on $A$ and $g \equiv 1$ on $X \backslash V$, we have $A=\left(f^{\prime}+g\right)^{-1}(0)$, as desired.

We remark that Theorem 1.5 of [RS], according to which each closed, $\sigma$-compact Baire set in a normal, locally compact space is a zero-set, follows from 1.5 above; for in general, any $\sigma$-compact subset of a locally compact space admits a $\sigma$-compact (hence, paracompact) neighborhood.

2. Contributions of $\boldsymbol{Z}$. Frolik. Theorem 1.2 above, and most of the results we have cited from [RS] (though not the [RS] results relating to measure and topological groups), can be deduced formally from the deep results pertaining to analytic sets codified and extended 
by Frolík in $\left[F_{2}\right]$ and in a sequence of papers summarized in $\left[F_{1}\right]$. Let us say, with Frolik, that the space $X$ is analytic if there is an upper semicontinuous multi-valued map $f$ from the space $\Sigma$ of irrational numbers onto $X$ which is compact (in the sense that $f(\sigma)$ is compact whenever $\sigma \in \Sigma$ ). Then each compact space, and each closed subspace of an analytic space, is analytic; thus each Baire set in an analytic space is analytic, hence Lindelöf. Now to prove Theorem 1.2, let $A$ be a closed Baire set in $X$, where $X$ is a Baire set in $\beta X$. Then $X$ is analytic, and $X \backslash A$ is a Baire set in $X$, hence is Lindelöf; like any open Lindelöf set in any space, $X \backslash A$ is a cozero-set (this uses the fact that the countable union of cozero-sets is a cozero-set). Thus $A$ is a zero-set in $X$.

Frolik has pointed out to the author, in connection with an early version of the present paper, that the proof in $\$ 1$ of 1.2 carries over directly to those sets which are (in his terminology) distinguishable from their complements. A formal definition runs as follows.

2.1. Definition. The subset $A$ of $X$ is said to be distinguishable in $X$ if there is a continuous function $\phi$ from $X$ into some separable metric space for which

$$
\phi(A) \cap \phi(X \backslash A)=\varnothing .
$$

The stronger version of 1.2 is, then, as follows.

2.2. Theorem. Let $A$ be a closed and distinguishable subset of $X$, and let $X$ be distinguishable in $\beta X$. Then $A$ is a zero-set in $X$.

For the proof, one shows as before that $A$ is distinguishable in $\beta X$, so that there is a continuous function $q$ from $\beta X$ to some metric space $M$ for which $q(A) \cap q(\beta X \backslash A)=\varnothing$. (The pair $(q, M)$ is constructed as follows. There are separable metric spaces $M_{1}$ and $M_{2}$, and continuous functions $\phi$ and $\psi$ from $X$ and $\beta X$ into $M_{1}$ and $M_{2}$ respectively, for which $\phi(A) \cap \phi(X \backslash A)=\psi(X) \cap \psi(\beta X \backslash X)=\varnothing$. Denoting by $M_{1}^{*}$ a metrizable compactification of $M_{1}$ and by $\phi^{*}$ the continuous extension of $\phi$ mapping $\beta X$ into $M_{1}^{*}$, we let $M=M_{1}^{*} \times M_{2}$ and $q(p)$ $=\left(\phi^{*}(p), \psi(p)\right)$ for $p$ in $\beta X$.) As above $q(A)$ is closed in $M$, hence has the form $f^{-1}(0)$ for some continuous, real-valued function on $M$, so that $A=(f \circ q)^{-1}(0)$, as desired.

In informal conversation, Frolik has suggested the likelihood that the concept of a distinguishable set may in the long run prove at least as fruitful and productive as the familiar Baire set concept. His forthcoming paper $\left[F_{2}\right]$ may serve to substantiate this suggestion. 


\section{REFERENCES}

[F $F_{1}$ Z. Frolik, A contribution to the descriptive theory of sets and spaces, Proc. Sympos. General Topology and its Relations to Modern Analysis and Algebra (Prague, 1961), Academic Press, New York, 1962, pp. 157-173. MR 26 \#3002.

$\left[\mathbf{F}_{2}\right]$ Zdenek Frolík, A survey of separable descriptive theory of sets and spaces, Czechoslovak Math. J. (to appear).

[GJ] Leonard Gillman and Meyer Jerison, Rings of continuous functions, The University Series in Higher Math., Van Nostrand, Princeton, N. J., 1960. MR 22 \#6994.

[H] Paul R. Halmos, Measure theory, Van Nostrand, Princeton, N. J., 1950. MR 11, 504.

$\left[\mathrm{N}_{1}\right]$ Stelios Negrepontis, Absolute Baire sets, Proc. Amer. Math. Soc. 18 (1967), 691-694. MR 35 \#4883.

$\left[\mathrm{N}_{2}\right] \longrightarrow$, Baire sets in topological spaces, Arch. Math. (Basel) 18 (1967), 603-608. MR 36 \#3314.

[RS] Kenneth A. Ross and Karl R. Stromberg, Baire sets and Baire measures, Ark. Mat. 6 (1965), 151-160. MR 33 \#4224.

Wesleyan University, Middletown, Connecticut 06457 